\title{
Sleep Oxygen Desaturation in Late Sequelae of Pulmonary Tuberculosis
}

\author{
Tetsuya Sakuma, Koichiro Tatsumi, Hiroshi Kimura, Yoshiyuki Honda* and Takayuki Kuriyama
}

Thirty-eight patients with late sequelae of pulmonary tuberculosis (TB seq.) were studied to clarify the characteristics of sleep desaturation in comparison with 40 patients with chronic obstructive pulmonary disease (COPD). While awake, the TB seq. group had a lower \% VC and a higher $\mathrm{PaCO}_{2}$. In both groups, the sleep lowest $\mathrm{SaO}_{2}$ was positively correlated with the awake $\mathrm{SaO}_{2}$. The regression line between the sleep lowest $\mathrm{SaO}_{2}$ and the awake baseline $\mathrm{SaO}_{2}$ in the $\mathrm{TB}$ seq. group was located below that in the $\mathrm{COPD}$ group. Awake $\mathrm{PaCO}_{2}$ was negatively related to the sleep lowest $\mathrm{SaO}_{2}$ only in the $\mathrm{TB}$ seq. group. These results indicate that the sleep lowest $\mathrm{SaO}_{2}$ values were lower in $\mathrm{TB}$ seq. than in $\mathrm{COPD}$ patients with the same levels of $\mathrm{SaO}_{2}$ while awake. Sleep studies are necessary to reveal the indication for nocturnal oxygen therapy in TB seq. patients, especially when they are hypercapnic in spite of their good awake oxygenation.

(Internal Medicine 35: 534-539, 1996)

Key words: chronic obstructive pulmonary disease, sleep study, home oxygen therapy

\section{Introduction}

Nocturnal oxyhemoglobin desaturation (NOD) has been documented in patients with various respiratory diseases such as chronic obstructive pulmonary disease (COPD) (1-3) and scoliosis $(4,5)$. COPD patients often manifest worsening $\mathrm{SaO}_{2}$ during sleep (1) and this nocturnal desaturation is reported to be ameliorated by supplemental oxygen inhalation with a beneficial effect in reducing pulmonary artery pressure (6). Therefore NOD has been incorporated in the indication for home oxygen therapy (HOT) since 1988 in Japan.

One of the characteristic features of HOT in Japan is its application for patients with late sequelae of pulmonary tuberculosis (TB seq.), which make up about one-fourth of the population receiving this therapy. In countries such as the United Kingdom and Japan, some patients who suffered from pulmonary tuberculosis in the prechemotherapy era are now suffering from respiratory failure (7-9). Most such patients underwent surgical treatments including lobectomy or thoracoplasty for collapsed lung to close the cavities. These surgical procedures limiting expansion of the lung have induced various thoracic deformities and, as a result, restrictive ventilatory impairments have developed. In patients with scoliosis with restrictive defects in respiratory functions, NOD has been reported to be more profound than in intrinsic lung disease, such as COPD (4). Because the patients with TB seq. have both intrinsic and extrinsic defects in the lungs, they may exhibit different characteristics of NOD in comparison with COPD. Accordingly, we attempted to compare the relationships between respiratory functions while awake and the degree of NOD in patients with TB seq. and COPD patients.

\section{Materials and Methods}

Forty patients with COPD and thirty-eight patients with TB seq. were studied. Anthropometric and pulmonary function data of both groups are shown in Table 1 . Chest X-ray examination was routinely performed on these patients. Patients with $\mathrm{FEV}_{1.0} / \mathrm{FVC}\left(\mathrm{FEV}_{1.0} \%\right)$ of less than $70 \%$, no past history of $\mathrm{TB}$ and no radiographic features of TB seq. were defined as COPD. TB seq. was specifically diagnosed on the basis of a past history of TB (culture verification of TB) and the radiographic features representing TB seq. such as thoracoplasty, pleural thickening, pulmonary resection and/or atelectasis, fibrosis, bronchiectasis, cavity formation and compensatory emphysematous change. The pulmonary function impairments were not uniform in the TB seq. group because of a variety of intrinsic and extrinsic pulmonary disorders. Eighteen of 38 patients with TB seq. had obstructive damage.

Most of the COPD and TB seq. patients were being treated

From the Department of Chest Medicine and *the Department of Physiology II, School of Medicine, Chiba University, Chiba

Received for publication December 18, 1995; Accepted for publication May 8, 1996

Reprint requests should be addressed to Dr. Tetsuya Sakuma, the Department of Chest Medicine, School of Medicine, Chiba University, 1-8-1 Inohana, Chuou-ku, Chiba 260 
Table 1. Anthropometric and Pulmonary Function Data

\begin{tabular}{lccc}
\hline & TB seq. & COPD & p Value \\
\hline Patients, $\mathrm{n}$ & 38 & 40 & \\
Sex, male:female & $30: 8$ & $34: 6$ & \\
Age, yr & $65 \pm 1$ & $64 \pm 2$ & NS \\
$\%$ ideal body weight & $89 \pm 3$ & $86 \pm 2$ & NS \\
$\%$ VC & $40 \pm 2$ & $66 \pm 2$ & $\mathrm{p}<0.01$ \\
$\mathrm{FEV}_{1.0} \%$ & $72 \pm 3$ & $49 \pm 2$ & $\mathrm{p}<0.01$ \\
$\mathrm{PaO}_{2}$, Torr & $71 \pm 1$ & $69 \pm 2$ & $\mathrm{NS}$ \\
$\mathrm{PaCO}_{2}$, Torr & $49 \pm 1$ & $43 \pm 1$ & $\mathrm{p}<0.01$ \\
$\mathrm{AaDO}_{2}$, Torr & $19 \pm 1$ & $28 \pm 2$ & $\mathrm{p}<0.01$ \\
\hline
\end{tabular}

Values are mean \pm SE.

with a wide variety of medications including theophylline derivatives, inhaled bronchodilators, and antimicrobials. These treatments were not interrupted throughout the study. The choice of our subjects was not concerned with sleep-related problems and none of them complained of such disorders. In fact, patients with sleep apnea syndrome were excluded from this study. At the time of the study, all subjects were in a stable clinical state, none having had an exacerbation within one month previously. Informed consent was obtained from all subjects.

Daytime arterial blood gases were taken while the patients were resting in the supine position, and pulmonary function tests were performed in the sitting position. Twenty-one out of 40 patients with COPD and 22 out of 38 patients with TB seq. underwent polysomnography in a quiet darkened room for two consecutive nights.

Electroencephalogram, electrooculogram, and submental electromyogram were recorded on a polygraph with sleep stages determined by the criteria of Rechtschaffen and Kales (10). We measured the rib cage and abdominal motions and nasal flow by an inductance plethysmograph and a thermistor, respectively, in all subjects. All subjects underwent continuous $\mathrm{SaO}_{2}$ monitoring for two consecutive nights, and the data showing the more severe desaturation was chosen for analysis. $\mathrm{SaO}_{2}$ was measured by finger-tip oximeter (Pulsox 7 or Biox II) connected to a microcomputer to record data every 1 or 2 seconds. Baseline $\mathrm{SaO}_{2}$ values were obtained in the supine resting position before sleep. We evaluated the degree of arterial oxygen desaturation during sleep by the lowest $\mathrm{SaO}_{2}$ and the total time spent with $\mathrm{SaO}_{2}$ of less than $85 \%$ (defined as $85 \%$ desaturation time). In the present study, we could only do polysomnography with some of the subjects because many patients with chronic respiratory disease complained of insomnia when we tried to perform it.

Because the angles of spinal deformity assessed by the method of Cobb in chest X-rays examination were small and had no relation to the degree of NOD, it was assumed unlikely that the presence of scoliosis accounted for the severe NOD in the TB seq. group.
Student's t-test was used for evaluating differences between the TB seq. and COPD groups. A least-square linear regression was used to evaluate the relationship between awake variables and sleep parameters. $\mathrm{p}<0.05$ was considered significant. All data were expressed as mean \pm SE.

\section{Results}

There was no difference between the two groups in age, $\%$ IBW and $\mathrm{PaO}_{2} . \mathrm{FEV}_{1.0} \%$ was significantly lower in the COPD group, while \% VC was considerably lower in the TB seq. group $(\mathrm{p}<0.01)$. The TB seq. patients had higher $\mathrm{PaCO}_{2}$ values than the COPD patients (Table 1).

Table 2 shows the analysis of the sleep study. There was no difference between the two groups in baseline $\mathrm{SaO}_{2}$. Although the $85 \%$ desaturation time was not different between the groups, the degree of sleep desaturation was more profound in the TB seq. group when evaluated by the lowest $\mathrm{SaO}_{2}$ values.

To exclude the influence of patients with lower baseline $\mathrm{SaO}_{2}\left(\mathrm{SaO}_{2}<90 \%\right)$ on our data, we further evaluated the differences between the two groups in patients with $\mathrm{SaO}_{2}$ $\geqq 90 \%$. The differences in all awake and sleep variables were statistically the same as those including all COPD and TB seq. patients.

In order to examine the effects of obstructive disorders on awake and sleep parameters in TB seq. patients, we compared these variables between the TB seq. patients with and without obstructive disorder. Although the patients with obstructive disorder had higher \%IBW (97 \pm 5 vs $82 \pm 3$ ), \%VC ( $47 \pm 3$ vs $36 \pm 2$ ) and larger $\mathrm{AaDO}_{2}(23 \pm 2$ vs $17 \pm 2)$, no differences were observed in sleep variables between the patients with and without obstructive impairment.

Mean sleep time of 21 patients with COPD and 22 patients with TB seq. was $362 \pm 15$ minutes (range 264-496 minutes) and $351 \pm 15$ minutes (range 268-442 minutes), respectively. We observed REM sleep in all COPD and TB seq. subjects at a mean of $75 \pm 5$ minutes and $75 \pm 6$ minutes, respectively. Maximal desaturation occurred during REM sleep in all subjects. Representative tracings of thoraco-abdominal movements by inductance plethysmography while awake and during sleep in one subject with TB seq. are shown in Fig. 1. There was no significant difference in the data of anthropometry, pulmonary function, arterial blood gases, sleep lowest $\mathrm{SaO}_{2}$ and sleep $85 \%$ desaturation time between the subjects tested and not tested by polysomnography in both the COPD and TB seq. groups.

Table 2. Sleep Data

\begin{tabular}{lccc}
\hline & TB seq. & COPD & p Value \\
\hline Baseline $\mathrm{SaO}_{2}, \%$ & $93 \pm 1$ & $92 \pm 1$ & NS \\
Sleep lowest $\mathrm{SaO}_{2}, \%$ & $76 \pm 2$ & $82 \pm 1$ & $\mathrm{p}<0.01$ \\
85\% desaturation time, min & $31 \pm 7$ & $49 \pm 17$ & NS \\
\hline
\end{tabular}

Values are mean \pm SE. 


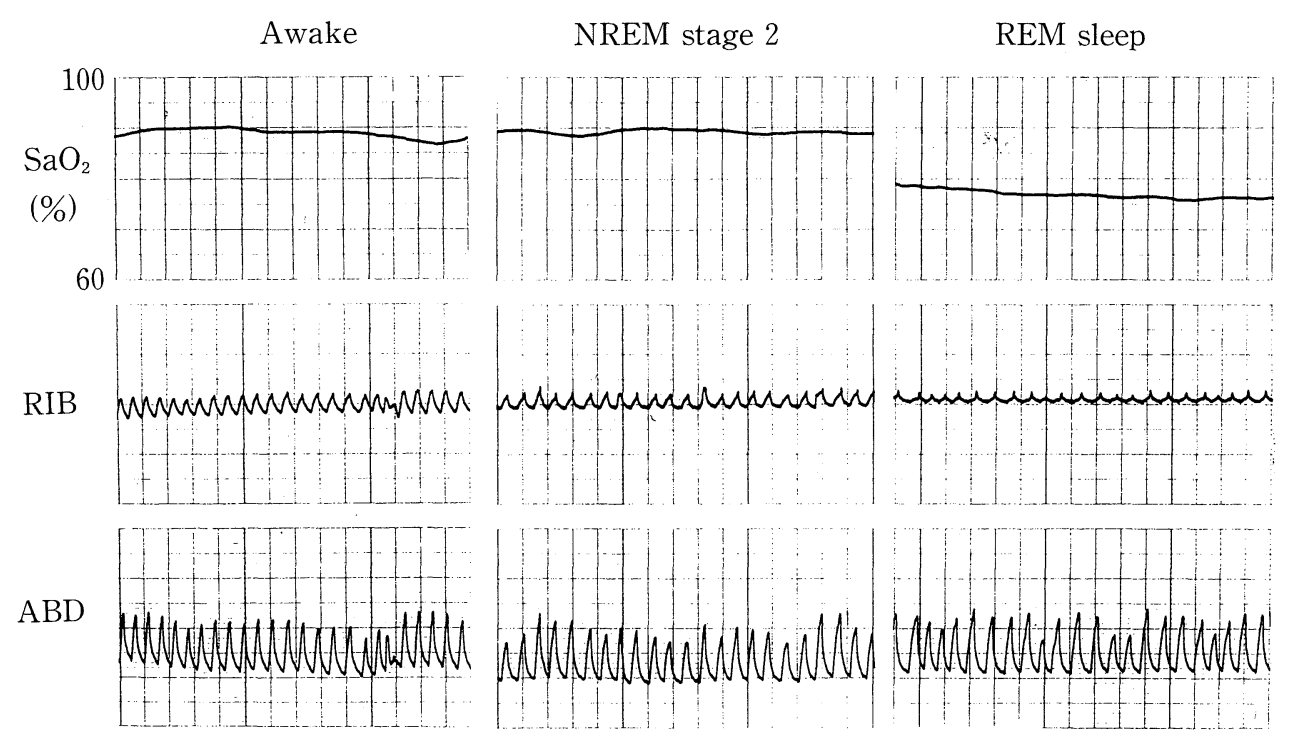

Figure 1. Representative tracing of thoraco-abdominal movements by Respitrace ${ }^{\circledR}$ while awake (left), during NREM sleep stage 2 (middle) and during REM sleep (right) in one subject with TB seq. NREM, non-rapid-eye-movement; REM, rapid-eye-movement; RIB, thoracic movement; ABD, abdominal movement.

To examine how daytime variables were correlated with the degree of NOD, we calculated the correlation coefficient of the linear regression analysis between the degree of NOD (the lowest $\mathrm{SaO}_{2}$ and $85 \%$ desaturation time) and daytime variables $\left(\mathrm{PaO}_{2}, \mathrm{PaCO}_{2}\right.$, baseline $\mathrm{SaO}_{2}, \mathrm{AaDO}_{2}, \mathrm{FEV}_{1.0} \%$ and \%VC) (Table 3). In the COPD group, baseline $\mathrm{SaO}_{2}$ or $\mathrm{PaO}_{2}$ was most closely related to the lowest $\mathrm{SaO}_{2}$ or $85 \%$ desaturation time. $\mathrm{AaDO}_{2}$ was also correlated with the degree of NOD. There was no significant relationship between $\mathrm{PaCO}_{2}$ and the degree of NOD. In the TB seq. group, however, $\mathrm{PaCO}_{2}$ was most closely related to the degree of NOD. Baseline $\mathrm{SaO}_{2}$ or $\mathrm{PaO}_{2}$ revealed a strong correlation with the degree of NOD, as was also the case in the COPD group. No correlation was observed between pulmonary function tests and the degree of NOD in either group.

The sleep lowest $\mathrm{SaO}_{2}$ plotted against baseline $\mathrm{SaO}_{2}$ is illustrated in Fig. 2. In the TB seq. group the regression line between these two variables was situated below that in the COPD group. In some of the TB seq. patients with baseline $\mathrm{SaO}_{2}>90 \%$, the sleep lowest $\mathrm{SaO}_{2}$ values were unexpectedly lower than those expected from the daytime $\mathrm{SaO}_{2}$ in $\mathrm{COPD}$ patients.

Figure 3 shows the relationship between awake $\mathrm{PaCO}_{2}$ and the sleep lowest $\mathrm{SaO}_{2}$ in both groups. There was a significant negative correlation between these two variables in the TB seq. but not in the COPD patients, indicating that the former patients with hypercapnia exhibited severe NOD.

The relationship between the sleep lowest $\mathrm{SaO}_{2}$ and $85 \%$ desaturation time is shown in Fig. 4. In each group there was a highly negative correlation between these two variables. The slpe of the regression line in the COPD group was steeper than in the TB seq. group, indicating that the duration of NOD was

Table 3. Correlation between Sleep Parameters and Awake Respiratory Variables in the Two Groups (Correlation Coefficients)

\begin{tabular}{|c|c|c|c|c|c|c|c|}
\hline \multirow[t]{2}{*}{ Sleep parameters } & \multirow[t]{2}{*}{ Group } & \multicolumn{6}{|c|}{ Awake respiratory variables } \\
\hline & & $\mathrm{PaO}_{2}$ & $\mathrm{PaCO}_{2}$ & Baseline $\mathrm{SaO}_{2}$ & $\mathrm{AaDO}_{2}$ & $\% \mathrm{VC}$ & $\mathrm{FEV}_{1.0} \%$ \\
\hline Sleep lowest $\mathrm{SaO}_{2}$ & $\begin{array}{l}\text { TB seq. } \\
\text { COPD }\end{array}$ & $\begin{array}{l}0.51 * \\
0.80 * * *\end{array}$ & $\begin{array}{l}-0.69 * * * \\
-0.23\end{array}$ & $\begin{array}{l}0.56 * * \\
0.85 * * *\end{array}$ & $\begin{array}{l}0.17 \\
-0.63 * * *\end{array}$ & $\begin{array}{l}0.22 \\
0.13\end{array}$ & $\begin{array}{l}-0.10 \\
0.03\end{array}$ \\
\hline $85 \%$ desaturation time & $\begin{array}{l}\text { TB seq. } \\
\text { COPD }\end{array}$ & $\begin{array}{l}-0.65^{* * *} \\
-0.62 * * *\end{array}$ & $\begin{array}{l}0.73 * * * \\
0.09\end{array}$ & $\begin{array}{l}-0.72 * * * \\
-0.86 * * *\end{array}$ & $\begin{array}{l}-0.10 \\
0.55^{* *}\end{array}$ & $\begin{array}{l}-0.07 \\
-0.01\end{array}$ & $\begin{array}{l}0.05 \\
-0.004\end{array}$ \\
\hline
\end{tabular}

${ }^{*} \mathrm{p}<0.005, * * \mathrm{p}<0.0005, * * * \mathrm{p}<0.00005$ 


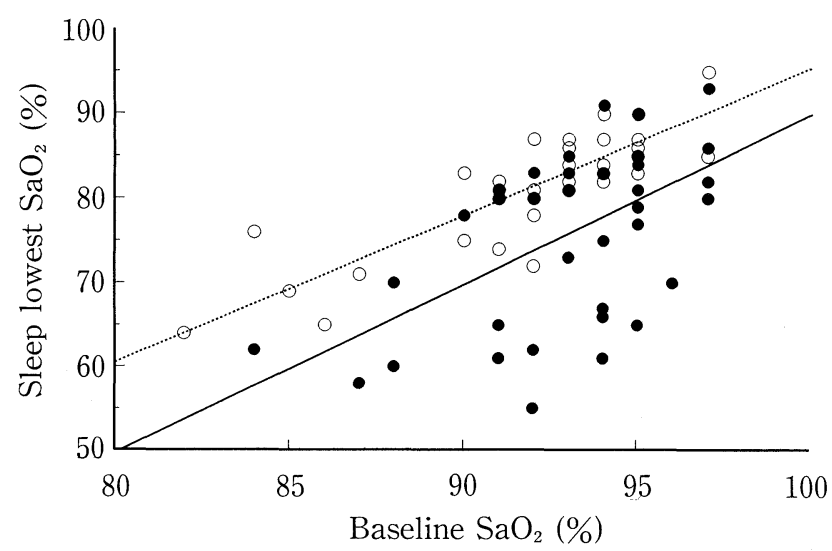

Figure 2. Relationship between baseline $\mathrm{SaO}_{2}$ and the sleep lowest $\mathrm{SaO}_{2}$ in the TB seq. group (closed circles with solid line) and the COPD group (open circles with broken line). The line represents linear regression in each group. The regression line of the TB seq. group was located below that of the COPD group. - - TB seq. $(n=38) y=2.0 x-111(r=0.56, p<0.01),--0-$ COPD $(n=40) y=$ $1.8 \mathrm{x}-79(\mathrm{r}=0.85, \mathrm{p}<0.01)$.

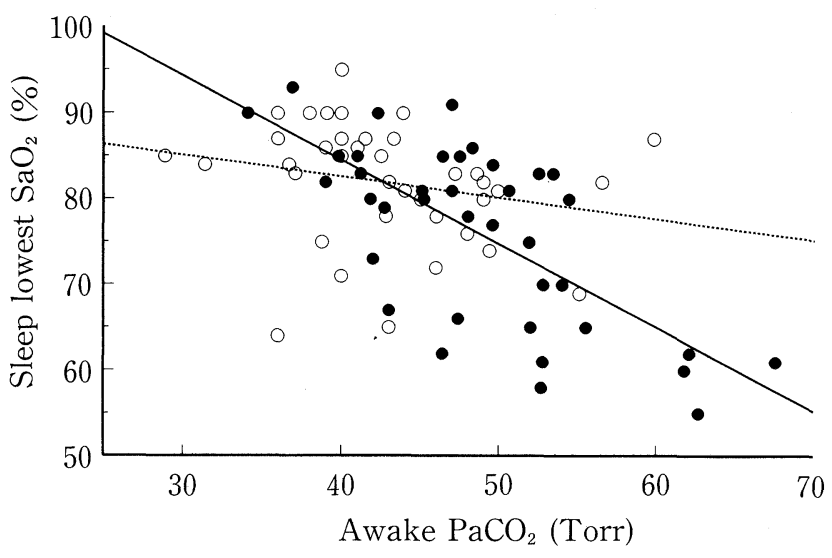

Figure 3. Relationship between awake $\mathrm{PaCO}_{2}$ and the sleep lowest $\mathrm{SaO}_{2}$ in the TB seq. group (closed circles with solid line) and the COPD group (open circles with broken line). The line represents linear regression in each group. $-\mathrm{TB}$ seq. $(\mathrm{n}=38) \mathrm{y}=$ $-0.98 x+124(r=-0.69, p<0.01),--0-$ COPD $(n=40) y=-0.25 x+93$ $(\mathrm{r}=-\mathbf{0 . 2 3}$, N.S.).

shorter in the TB seq. patients in spite of the lower nadir $\mathrm{SaO}_{2}$.

\section{Discussion}

TB seq. is now well recognized as one of the diseases for which HOT can be provided in Japan. Twenty percent of the patients receiving HOT were TB seq. in 1994. Before the 1960s there was hardly any effective treatment for tuberculosis, because chemotherapy was not yet in widespread use. In those days, surgical treatments such as partial resection of several ribs (thoracoplasty) were often carried out to collapse air spaces in

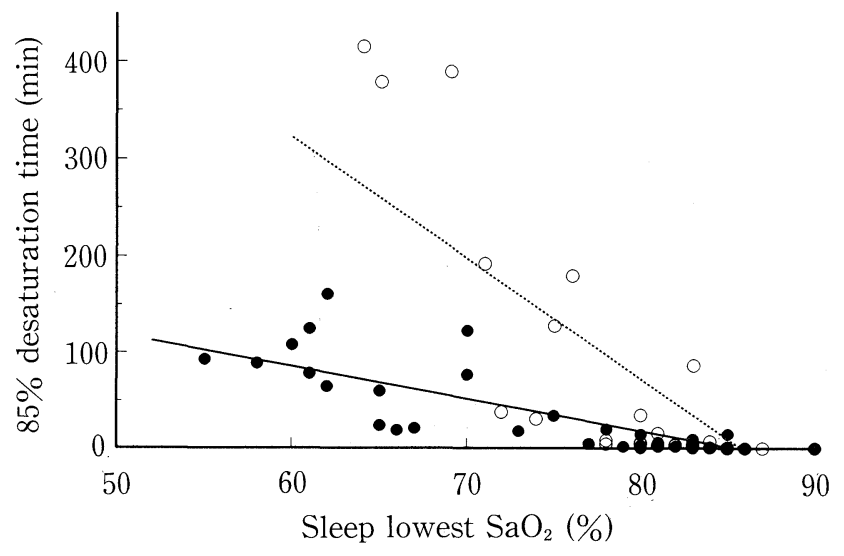

Figure 4. Relationship between the sleep lowest $\mathrm{SaO}_{2}$ and $85 \%$ desaturation time (total sleep time with $\mathrm{SaO}_{2}$ less than $85 \%$ ) in the TB seq. group (closed circles with solid line) and the COPD group (open circles with broken line). The line represents linear regression in each group. $\rightarrow$ TB seq. $(n=38) y=-3.4 x+290(r=$ $-0.80, p<0.01),--\bigcirc-$ COPD $(n=40) y=-12.5 x+1,070 \quad(r=-0.79$, p<0.01).

the infected lung. These procedures caused mechanical disadvantages in the chest cage, which later resulted in deteriorated respiratory function with restrictive impairment. Lung compliance decreased because of postinfectious lung fibrosis and pleural thickening and because of the loss of lung volume secondary to thoracoplasty. Chest wall compliance fell because of progressive fibrosis of the soft tissues of the rib cage as well as thoracic scoliosis. Some of the patients seem to have gradually developed respiratory failure after thoracoplasty (7-9). In TB seq. patients, hypoxemia seems to be mainly due to alveolar hypoventilation, because an increase in $\mathrm{PaCO}_{2}$ with nearly normal $\mathrm{AaDO}_{2}$ (gas exchange) generally develops. This late appearance of chronic hypercapnia may be explained by decreased lung volume, and decreases in respiratory drive with age, chest wall compliance and inspiratory muscle strength, and by respiratory muscle fatigue.

In this study, we have clarified the characteristics of sleep desaturation in TB seq. patients as compared with COPD patients. We found that NOD occurred in most TB seq. patients, as already reported in COPD patients, and that the magnitude of NOD was more profound in TB seq. patients even though they had the same levels of awake baseline $\mathrm{SaO}_{2}$. In addition, we demonstrated that the values of baseline $\mathrm{SaO}_{2}$ and $\mathrm{PaCO}_{2}$ while awake were correlated with the degree of NOD in TB seq. patients. These results indicate that TB seq. patients with lower oxygen saturation and lower alveolar ventilation in wakefulness tend to be predisposed to severe NOD.

Previous studies have demonstrated that many COPD patients have frequent and prolonged episodes of hypoxemia during sleep (11), as we also found in this study. It is likely that a major cause of these nocturnal hypoxemic episodes is hypoventilation, although other mechanisms such as further impairment of ventilation-perfusion mismatch and upper airway obstruction may contribute to the nocturnal desaturation. 
In the present study, the feature of NOD in TB seq. patients was different from that in COPD. Nocturnal desaturations in TB seq. consisted of frequent short-duration episodes with striking hypoxemia. In TB seq. patients, a decrease in functional residual capacity, which reflects a decrement in $\% \mathrm{VC}$, may be involved in the profound degree of sleep desaturation observed, based on the analogy of the findings that initial lung volume is an important determinant of hypoxemia during apnea (12). However, because \%VC was not significantly correlated with the magnitude of sleep desaturation in this study, other factors may also have contributed to the different pattern of NOD from that in COPD.

In the present study, we found that the magnitude of nocturnal hypoxemia was closely related to the values of awake $\mathrm{PaCO}_{2}$ in TB seq. patients, but no such relationship was observed in COPD patients. This may be partly explained by the fact that our COPD patients were less hypoxic and less hypercapnic while awake than TB patients. It may be that the stage of disease in our COPD subjects was relatively mild. PerezPadilla et al (13) reported that hypercapnic COPD had more severe NOD than normocapnic patients. On the other hand, some TB seq. subjects had unexpectedly severe NOD even though their awake $\mathrm{PaO}_{2}$ was above 70 Torr. Such patients would not be indicated for HOT because of their good awake oxygenation. It should be recognized that hypercapnic TB seq. patients have more severe NOD compared with advanced COPD patients with severe awake hypoxemia.

In TB seq. patients, NODs were predominantly observed during REM sleep. Accessory respiratory muscles are known to support ventilation while awake in TB seq. patients. REM sleep-related muscular atonia and blunted ventilatory responses may produce greatly suppressed thoracic respiratory movements with unaffected abdominal movements. These abnormalities may result in a combination of alveolar hypoventilation and a worsening of gas exchange, which produces severe sleep desaturation during REM sleep.

The mechanisms for short and repetitive NOD observed in TB seq. patients were considered as follows. Ventilatory drive augmented by hypoxic-hypercapnic interaction may induce arousal response which terminates an NOD episode. The magnitude of this chemical ventilatory drive in TB seq. patients may be relatively strong because the initial awake $\mathrm{PaCO}_{2}$ is already high and the rate of $\mathrm{PaCO}_{2}$ increase may be largely due to a possible decrease in the alveolar space as mentioned above. On the other hand, the duration and degree of NOD in COPD patients may be largely determined by hypoxic ventilatory drive due to the lower initial $\mathrm{PaCO}_{2}$ level and an expected lower rate of $\mathrm{PaCO}_{2}$ increase. The lesser contribution of $\mathrm{CO}_{2}$ drive may be related to the longer NOD in COPD patients.

Repetitive episodes of transient hypoxemia during sleep may cause an increase in pulmonary vascular resistance (1416). Midgren et al (17) reported that 5 of their 8 scoliotic patients with nocturnal hypoxemia had suffered from right heart failure at least once, and that 7 had been hospitalized because of respiratory problems. In addition, according to the definition proposed by Fletcher et al (18) (decrease in $\mathrm{SaO}_{2}$ for at least 5 minutes to or below $85 \%$ during sleep), the survival rate was only $50 \%$ in 38 COPD patients after 4 years, vs $80 \%$ in the nondesaturated group $(\mathrm{p}<0.02)$. We therefore believe that supplemental oxygen during sleep is indicated in patients with TB seq. showing repetitive NOD.

In summary, the wide gap between awake hypoxemia and NOD seems to be mainly caused by the deleterious effects of sleep on the ventilatory muscle pump. This feature is explicit in thoracic constrictive lung diseases such as TB seq. and scoliosis. The present study suggests that a sleep study is indispensable for detecting unpredictably severe NOD in thoracic constrictive lung diseases, and in that case the best indicator is the awake $\mathrm{PaCO}_{2}$ level.

Acknowledgements: This study was supported in part by a grant from the Research Committee, Intractable Respiratory Failure, the Ministry of Health and Welfare of Japan, and in part by a grant from the Chiba Dispensary of the Japan Anti-tuberculosis Association.

\section{References}

1) Wynne JW, Block AJ, Hemenway J, Hunt LA, Flick MR. Disordered breathing and oxygen desaturation during sleep in patients with chronic obstructive lung disease (COLD). Am J Med 66: 573, 1979.

2) Douglas NJ, Calverley PMA, Leggett RJE, Brash HM, Flenley DC, Brezinova V. Transient hypoxaemia during sleep in chronic bronchitis and emphysema. Lancet 1: 1, 1979.

3) Koo KW, Sax DS, Snider GL. Arterial blood gases and pH during sleep in chronic obstructive pulmonary disease. Am J Med 58: 663, 1975.

4) Midgren B. Oxygen desaturation during sleep as a function of the underlying respiratory disease. Am Rev Respir Dis 141: 43, 1990.

5) Mezon BL, West $P$, Israels J, Kryger $M$. Sleep breathing abnormalities in kyphoscoliosis. Am Rev Respir Dis 122: 617, 1980.

6) Weitzenblum E, Sautegeau A, Ehrhart M, Mammosser M, Pelletier A. Long-term oxygen therapy can reverse the progression of pulmonary hypertension in patients with chronic obstructive pulmonary disease. Am Rev Respir Dis 131: 493, 1985.

7) Phillips MS, Kinnear WJM, Shneerson JM. Late sequelae of pulmonary tuberculosis treated by thoracoplasty. Thorax 42: 445, 1987.

8) Zimmermann HA. Hemodynamics: studies on a group of patients who developed cor pulmonale for thoracoplasty. J Thoracic Surg 22: 94, 1951.

9) Sawicka EH, Branthwaite MA, Spencer GT. Respiratory failure after thoracoplasty: treatment by intermittent negative pressure ventilation. Thorax 38: 433, 1983.

10) Rechtschaffen A, Kales A, eds. A Manual of Standardized Terminology, Techniques and Scoring System for Sleep Stage of Human Subjects. (NIH publication 204). National Institutes of Health, Bethesda, 1968.

11) Tatsumi K, Kimura H, Kunitomo F, Kuriyama $T$, Watanabe $S$, Honda $Y$. Sleep arterial oxygen desaturation and chemical control of breathing during wakefulness in COPD. Chest 90: 68, 1986.

12) Findley LJ, Ries AL, Tisi GM, Wagner PD. Hypoxemia during apnea in normal subjects: mechanisms and impact of lung volume. J Appl Physiol 55: 1777, 1983.

13) Perez-Padilla R, Conway W, Roth T, Anthonisen N, George C, Kryger M. Hypercapnia and sleep $\mathrm{O}_{2}$ desaturation in chronic obstructive pulmonary disease. Sleep 10: 216, 1987.

14) Nattie EE, Doble EA. Threshold of intermittent hypoxia-induced right ventricular hypertrophy in the rat. J Appl Physiol 56: 253, 1984.

15) Doyle JT, Wilson JS, Warren JV. The pulmonary vascular responses to short-term hypoxia in human subjects. Circulation 5: 263, 1952.

16) Fletcher EC, Luckett RA, Miller T, Costarangos C, Kutka N, Fletcher JG. Pulmonary vascular hemodynamics in chronic lung disease patients with and without oxyhemoglobin desaturation during sleep. Chest 95: 757, 
Sleep Desaturation in Late Sequelae of Pulmonary Tuberculosis

1989.

17) Midgren B, Petersson K, Hansson L, Eriksson L, Airikkala P, Elmqvist D. Nocturnal hypoxaemia in severe scoliosis. Br J Dis Chest 82: 226, 1988.
18) Fletcher EC, Miller J, Divine GW, Fletcher JG, Miller T. Nocturnal oxyhemoglobin desaturation in COPD patients with arterial oxygen tensions above $60 \mathrm{mmHg}$. Chest 92: 604, 1987. 